\title{
TLS FOR DETECTING SMALL DAMAGES ON A BUILDING FAÇADE
}

\author{
A. Masiero ${ }^{\mathrm{a}, *}$, D. Costantino ${ }^{\mathrm{b}, \mathrm{c}}$ \\ ${ }^{\text {a }}$ Interdepartmental Research Center of Geomatics (CIRGEO), University of Padova, \\ Viale dell’Università 16, Legnaro (PD) 35020, Italy - masiero@dei.unipd.it \\ ${ }^{\mathrm{b}}$ DICATECh, Polytechnic of Bari, Italy, 70126 Bari, Italy - domenica.costantino@ poliba.it \\ ${ }^{\mathrm{c}}$ Faculty of Engineering, Salento University, 73100 Lecce, Italy - domenica.costantino@unisalento.it
}

KEY WORDS: Laser Scanning, Damage Detection, Maintainance, Spatial Filtering

\begin{abstract}
:
Weathering, aging, infiltration, solar radiation and several other factors cause the deterioration of buildings and infrastructures and hence the need for periodical maintenance and restoration. The need for maintenance has been traditionally determined based on visual inspections of qualified operators. Since this process is obviously time consuming and quite expensive, especially when the considered building is quite large and high, then a number of recent studies have been recently published proposing remote sensing tools in order to ease the monitoring process. Among the possible spatial data acquisition sensors, terrestrial laser scanning has been considered in several of the existing studies, mostly because of its high reliability, to cope with cracks and defect detection up to the millimeter level of resolution, which is the typical accuracy of the current generation of professional laser scanners. This paper considers the problem of detecting small defects on the façade of a University building. Similar to other previous studies, in this work defect detection is accomplished by considering distances with respect to a planar surface locally fitted on the building façade. Then, statistical filtering and machine learning tools have been implemented in order to cope with damage detection of the brick surfaces at sub-millimeter level.
\end{abstract}

\section{INTRODUCTION}

Aging, humidity, infiltration, solar radiation and other physical, chemical and biological factors cause the need for monitoring building structures and their periodical restoration (Giacomucci et al., 2011. Hällström et al., 2009, Sextos et al., 2018). Monitoring the status of building façades is a task typically done by a human operator (Bauer et al., 2014), which has to carefully inspect them in order to determine areas that require restoration or maintenance.

Despite careful human inspection ensures very good results when dealing with easily visible parts of the façade (e.g. close to the ground), it may be unreliable, subjective (or expensive) for areas on the top of the façade, which are typically hard to be clearly checked by the operator. In practice, a fast and reliable assessment of the damaged areas on a relatively large façade may be not so easy for a human operator.

The above considerations motivate the use of alternative, mostly automated, remote sensing tools for checking damaged areas on a building façade. To be more specific, this work considers the problem of determining damaged bricks on a building of the University of Padua (Fig. 11. Given the significant amount of damaged bricks on such façade it is very important to determine a reliable estimate of the number of those to be restored/substituted in order to limit the current maintenance costs while ensuring a satisfying result.

The rationale of this work is that of using typical geomatics instruments and tools, such as terrestrial laser scanning and spatial statistical filtering, in order to develop an easy to use and quite fast method to determine damaged bricks on a building façade (Fig. 1] and 2).

Given the different aspect of damaged bricks with respect to regular ones (Fig. 2), a viable way to detect damaged bricks can

\footnotetext{
${ }^{*}$ Corresponding author.
}

be that of using images exploiting the recently developed neural networks recognition techniques (e.g. deep learning). Image acquisition from UAV (in order to use close-view images) can be particularly well suited in combination with such techniques ((Galarreta et al., 2015, Alicandro et al., 2018)) or to ease the visual inspection (Angeli et al., 2018). Photogrammetric techniques have also been considered, also in combination with thermography, for structural investigations (Costantino and Angelini, 2012. Masiero et al., 2015) and in order to assess and characterize detachments on façades (Maierhofer et al., 2015).

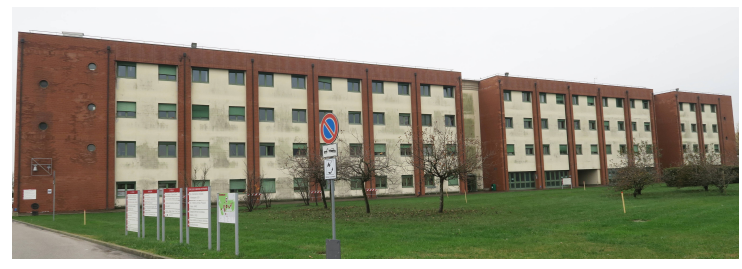

Figure 1. University building façade considered in this work.

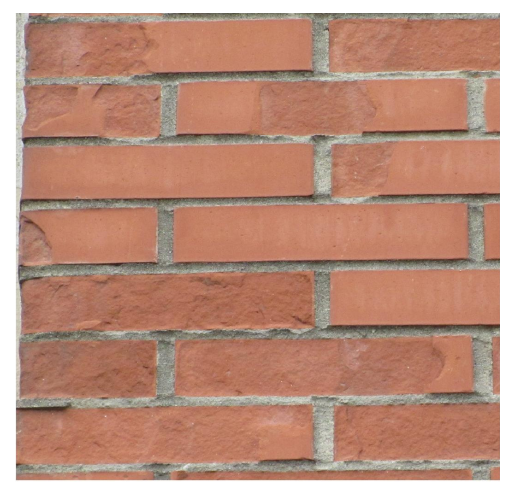

Figure 2. Close view of bricks on the façade. 
Despite image based analysis can lead to interesting results, it may fail in correctly distinguishing slightly damaged bricks from dirty ones, because a chromatic alteration of brick appearance is associated to both such cases. A similar consideration applies also to the usage of intensities of laser scanning measurements, which can also be considered for structure monitoring (ArmestoGonzález et al., 2010. Hemmleb et al., 2006).

Motivated by this consideration, in this work terrestrial laser scanning (TLS) is used to produce a very accurate description of the façade. Then, brick damage detection is done based on a geometric analysis of the acquired information.

Actually, TLS have already been considered in several works for damage and cracks detection and structural analysis of infrastructures and building façades (Almac et al., 2016 Tucci et al., 2016. Guarnieri et al., 2017b Suchocki and Błaszczak-Bk, 2019. Guarnieri et al., 2017a). Similarly to most of such previous works on damage detection with TLS, the basic principle of geometric damage detection is that of checking for variations with respect to a locally fitted (typically planar) surface (Teza et al., 2009 Kim et al., 2014 Bosché and Guenet, 2014, Wang et al., 2016. AlNeshawy et al., 2009). However, defects and damages considered in previous works typically were at millimeter level, whereas in this work the depth difference between a damaged brick surface and one in good conditions can be at sub-millimeter level. Since the variations/damages of interest in this work can be smaller than the typical sensor accuracy, the acquired data should be properly processed in order to enhance the quality of their geometric information.

The paper is organized as follows: an overview of the proposed workflow is presented in Section 2. Then, details about the processing procedure are provided in Sections 3. and 4. Some results are shown in Section 5. and, finally, discussion and concluding remarks are reported in Section 6 .

\section{PROCEDURE OVERVIEW}

The proposed procedure is based on the analysis of high resolution TLS scans of a building façade. Scans have been acquired by using a Leica ScanStation C10, whose nominal position and distance accuracies are (at least) $6 \mathrm{~mm}$ and $4 \mathrm{~mm}$ for $1-50 \mathrm{~m}$ ranges. The scanner was positions were choosen according to the following criteria:

- ensure a sufficiently small range measurement error,

- ensure incident angles quite close to perpendicular to the façade surface,

- reduce the number of required scans.

According to the above criteria, the scanner was positioned at approximately $27 \mathrm{~m}$ from the façade (which is approximately $16 \mathrm{~m}$ high) and acquisitions was limited to the portion of the façade just in front of the scanner.

Brick damage detection on each area of the façade is done by considering just the scan with the best combination of closenessinclination angle, consequently the registration between different scans is only done for avoiding to repetitively analyze the same area: the use of standard registration techniques, for instance by using targets, allows to obtain a sufficient level of registration accuracy between different scans, hence the procedure description provided in the following will focus just on the analysis of a scan.
Each brick is detected and segmented in the point cloud corresponding to the currently considered scan, as described in Section 3. In this work, brick segmentation is based on the analysis of the measured laser intensities and on the prior information available on the brick shapes.

Then, statistical smoothing is applied to each brick point cloud in order to reduce the measurement noise and obtain reliable information on the brick geometry (Section 4.).

Finally, certain suitable statistics are extracted from each brick and used as input for a properly trained machine learning classifier that is used to detect damaged bricks.

\section{BRICK SEGMENTATION}

Given the high level of regularity of the brick disposition on the façade of interest, it is quite clear that the brick segmentation procedure can take advantage from such regularity.

First, brick areas of the façade can be roughly determined by setting a proper threshold on the measured laser intensity. Then, the planar surface which fits best the considered brick area on the façade is computed and exploited in order to change the TLS point cloud reference system: $x$ and $y$ axes are set on the detected plane, with $x$ and $y$ axes corresponding to the horizontal and vertical direction, respectively. $z$ axis is set orthogonal to façade surface, pointing outside of the wall (e.g. towards the laser scanner, approximately, as shown in Fig. 3.

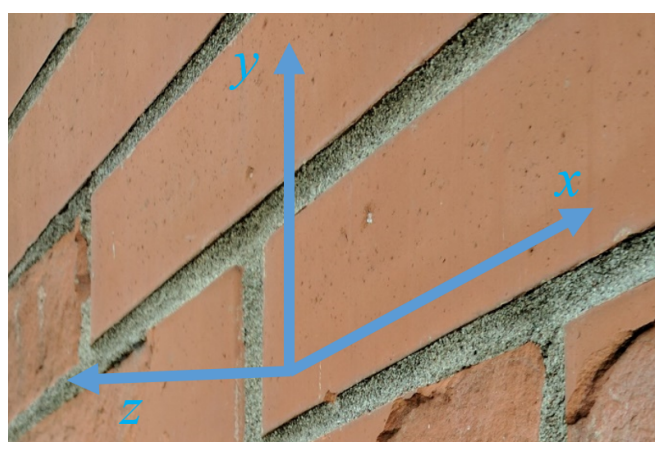

Figure 3. Reference system.

Then, similarly to (Sithole, 2008), a rough brick segmentation can be obtained by taking into account of the laser intensities and of the regular brick pattern, as shown Fig. 4

Finally, a more precise single brick segmentation is obtained by detecting the best rectilinear contour lines (each line shall be either parallel or orthogonal with each other one of the same brick) according to the nominal brick sizes: actually, in order to ensure some flexibility to the developed method, the brick sizes are required to be within a maximum error threshold with respect to the nominal values (the maximum threshold is set to $0.5 \mathrm{~cm}$ in the experimental results shown in this paper).

\section{STATISTICAL FILTERING OF BRICK POINT CLOUDS}

Since the goal of this work is that of being able to detect even small damages and detachments on bricks, the considered procedure shall be able to detect sub-millimeter changes along the $z$-direction of each brick (most of the small damages are at such level). 


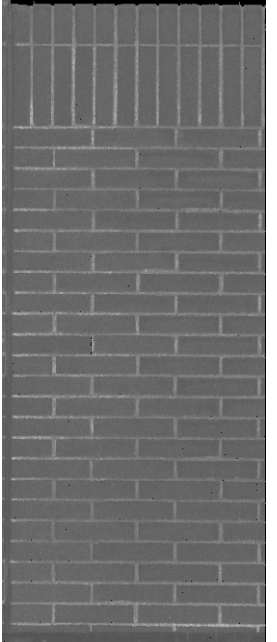

(a)

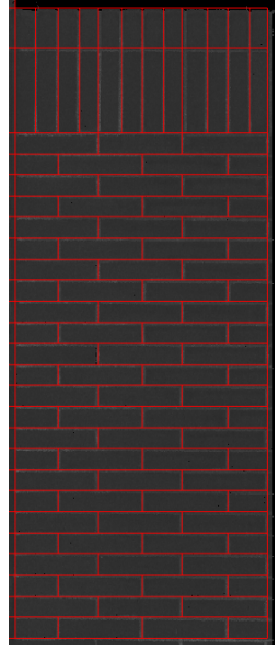

(b)
Figure 4. (a) Laser intensities (a) and (b) rough brick segmentation.

First, assume to consider a brick in good conditions (e.g. Fig.5 (5)) and to locally fit a planar surface to its point cloud. Then, compute the distribution of the point-to-plane distances, as shown in Fig. 6. a). Since the considered brick is good conditions, the planar surface should well describe the real geometry of the brick, hence the distribution shown in Fig. 5(a) can be considered as quite representative of the a posteriori laser scanning measurement error (standard deviation of $1.7 \mathrm{~mm}$ for the distribution in Fig. 5(a)), or, more precisely, it can be used to assess the measurement error statistical characteristics along the direction orthogonal to the brick surface, which actually is the direction our main interest in order to discriminate damaged bricks.

Then, the above procedure is repeated for the damaged brick shown in Fig. 5(b), obtaining the error distribution in Fig. 6b) ((standard deviation of $2.2 \mathrm{~mm})$.

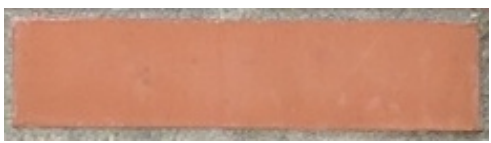

(a)

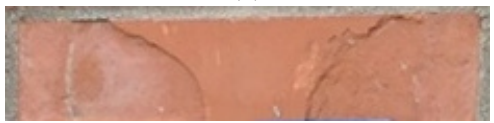

(b)

Figure 5. Example of brick in good conditions (a) and a damaged one (b).

As shown in Fig. 6 roughness/geometric variations in the two considered studies are quite hardly visible by comparing the two point-to-plane distance distributions: despite a slight difference in the standard deviation, the two distributions are quite similar. Given such similarity, it is quite apparent that in both the cases the (approximately Gaussian) shape of such distributions is mostly caused by the laser scanner measurement noise: actually, in most of the considered bricks, laser scanning measurement noise is larger than the typical "depth" of the damages to be detected.

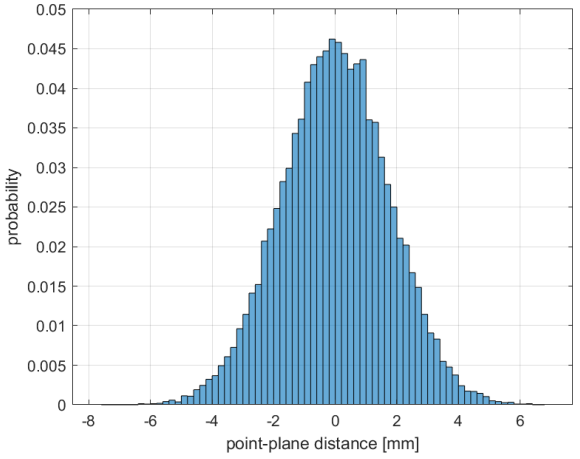

(a)

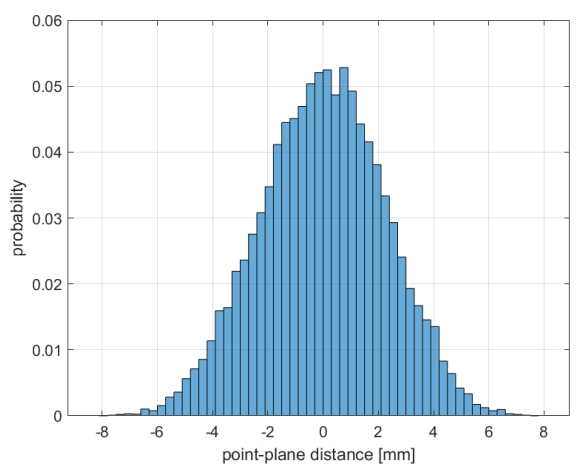

(b)

Figure 6. Histograms of point-to-plane distances for the two bricks shown in Fig. 5

\subsection{Measurement error and spatial filtering}

The simplest measurement error model that can be formulated is that obtained by modeling the measurements as affected by a zero-mean independent and identically distributed noise, with standard deviation $\sigma$.

According to such model, if $n$ measurement samples are available on the same point their average can be used in order to reduce the uncertainty on the signal value to $\sigma / \sqrt{n}$.

Since $\sigma \approx 2 \mathrm{~mm}$ and the goal is that of distinguishing depths at sub-millimeter level, then $n$ should be chosen in such a way to ensure for instance $\frac{\sigma}{\sqrt{n}} \approx 0.2 \mathrm{~mm}$, hence $n \approx 100$.

In practice, collecting such a large number of TLS measurement samples (approximately) on the same position entails a lot of acquisition time, hence it is impracticable way. Actually, spatial sample averaging can be used instead of time sample averaging to cope with the acquisition time issue.

Obviously, since spatial averaging reduces the spatial resolution of the filtered signal a high density of points shall be available in order to ensure a sufficient spatial resolution of the obtained result.

Furthermore, the considered error model only describes only approximately the real error. Consider for instance the case of the brick in Fig. 5 (a), and assume to filter the $z$ values averaging the measured values in a $1 \mathrm{~cm}$ square neighborhood (which corresponds in the considered case to an average number of considered points $n=165$ ): the resulting distribution, shown in Fig. 7.a) has standard deviation $0.025 \mathrm{~mm}$. Since such computation has 
been done on a brick in good conditions the obtained error on the average estimates shall be due to the measurement error: the difference between the theoretical value of the standard deviation of the average and the real one shall indicate the presence of correlation on such noise, which can be estimated and exploited to improve the spatial filtering, leading to a Kriging-like approach.

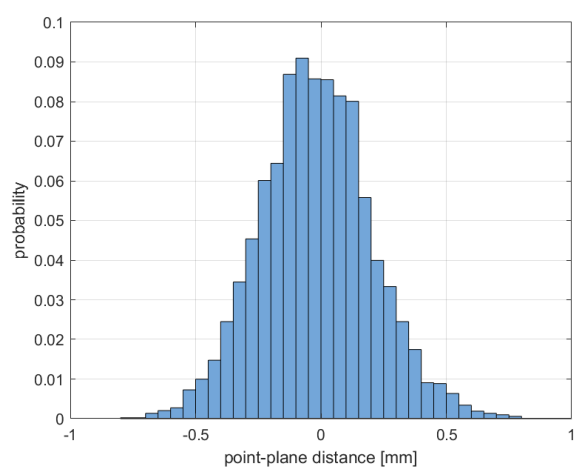

(a)

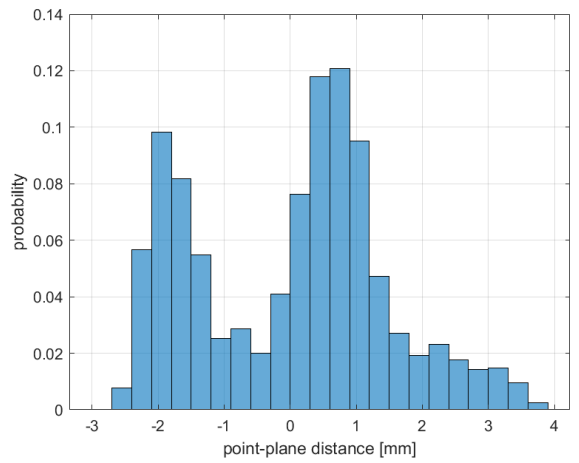

(b)

Figure 7. Histogram of point-to-plane distances for damaged brick shown in Fig. 5. b).

In order to check the effectiveness of the spatial filtering procedure for the detection of small damages on the bricks, Fig. 7 b) shows the error distribution of the filtered results obtained on the brick shown in Fig. 5(b). Differently from Fig. 7 a), it is clear that in this case most of the variation along the $z$ axis is due to the damage: it is actually apparent that the distribution in Fig. 7 b) is multi-modal, where the two modes are associated to the central (in good conditions) and to the left and right (damaged) areas of the brick in Fig. 5(b).

\subsection{Outlier rejection}

Outliers laser measurements and the presence of thin objects, such as cables, in front of a brick may have a large impact on the extraction of reliable statistics about such brick. A simple outlier rejection step is implemented in order to reduce the issues related to such kind of samples.

The distribution of the filtered $y$ components of a brick in good conditions are assumed to be centrally distributed, and, in particular, approximately Normally distributed. The median absolute deviation is used as a robust estimator of the standard deviation of such distribution, properly scaled by a multiplicative factor $\approx 1.48$.

Then, measurement samples at distances from the fitting plane larger than three times the estimated standard deviation are considered as outliers and discarded from the analysis procedure.

\subsection{Brick classification}

Several statistics have been considered on the filtered $z$ in order to be used as inputs for a support vector machine (SVM) classifier, which has been trained on 100 bricks.

Actually, best results have been obtained by using the following as inputs for the classifier:

- the roughness index (the standard deviation on the $z$ ),

- the fraction between the roughness before and after the filtering procedure.

\section{RESULTS}

The proposed procedure has been applied on a set of 523 bricks on the top part of the building façade, with the laser scanner positioned on the ground, approximately $27 \mathrm{~m}$ far from the façade. Most of the test area is shown in Fig. 8 TLS acquisitions have been done with a high point density, $120-130$ points $/ \mathrm{cm}^{2}$.

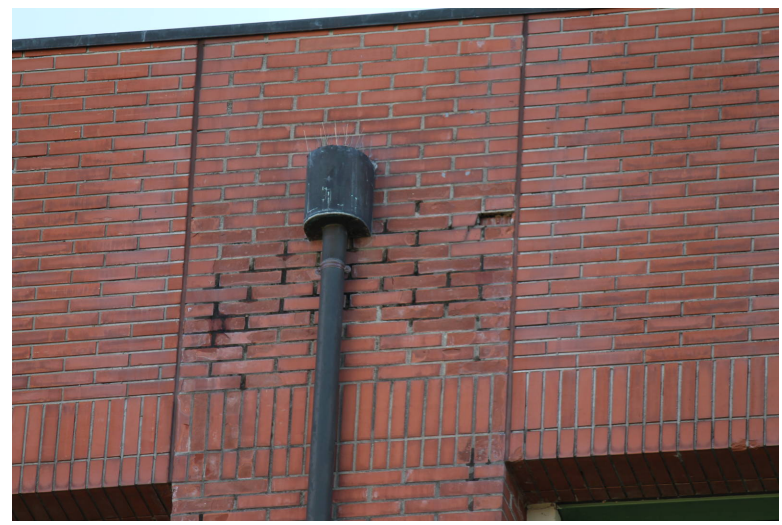

Figure 8. Test area.

Fig.9 9 shows the roughness index of the filtered point cloud comparing the roughness of the bricks in good conditions (blue) with respect to the damaged ones (red).

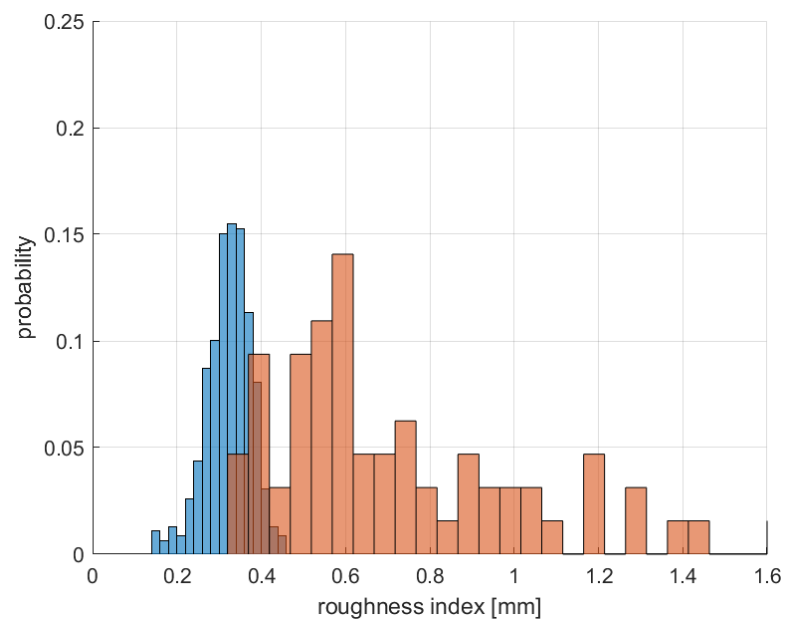

Figure 9. Comparison of roughness index distributions for bricks in good conditions (blue) and damaged ones (red).

Fig. 10 shows the misclassified bricks (in red): 9 bricks were misclassified (1.7\% error). 


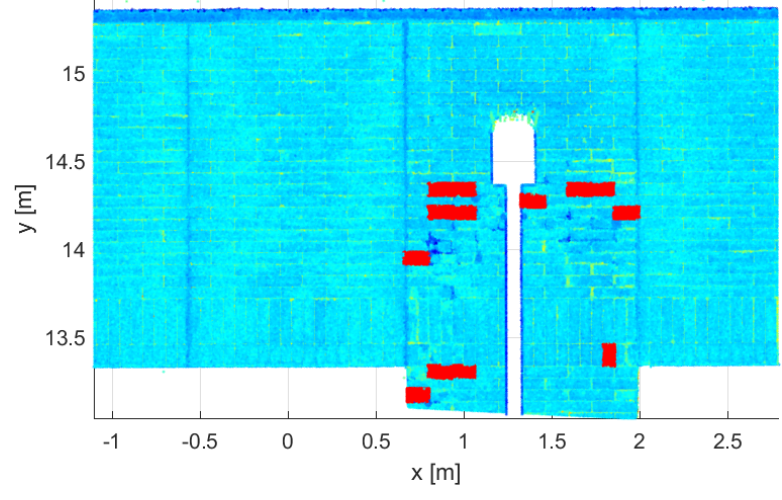

Figure 10. Misclassified bricks (red).

Fig. 10 shows an example of misclassified damaged brick: despite the brick is damaged, its surface is quite close to planar, making it hard to be distinguished with respect to one in good conditions.

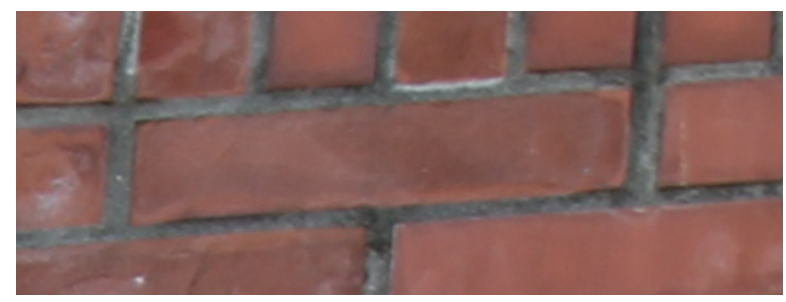

Figure 11. Example of misclassified brick.

\section{CONCLUSIONS}

The proposed procedure, based on spatial filtering in order to reduce the TLS random measurement error and then on machine learning (SVM) classification to detect damaged bricks, allowed to obtain sub-millimeter accuracy in the description of the $z$ coordinate (i.e. depth of the damage, if any) of the brick 3D points.

It is worth to notice that, since only points within a single brick are simultaneously analyzed in the detection procedure, actually TLS systematic error has a minor effect. Nevertheless, such effect is visible on the factor between the roughness index before and after spatial filtering: since such factor is smaller then expected by theory when considering independent samples, some correlation between errors on the same brick is probably present, and, if properly modeled, might be exploited in order to further improve the system performance. This aspect will be considered in our future works.

The proposed procedure allowed to correctly classify $98.3 \%$ of the bricks, where most of the errors were related to damaged bricks with almost planar surfaces, as shown in Fig.11

Despite the proposed procedure is basically exploiting only the 3D information of the acquired dataset, the 3D geometry can be combined with the measured laser intensity in order to develop an approach exploiting all the information provided by the TLS measurements. The introduction of more artificial intelligence recognition tools (Simonyan and Zisserman, 2014, Pierdicca et al., 2018), such as those based on convolutional neural networks, will also be considered in our future research work in order to improve the system performance.

\section{REFERENCES}

Al-Neshawy, F., Erving, A. and Puttonen, J., 2009. Measuring the bowing of marble panels in building facades using terrestrial laser scanning technology. Journal of Information Technology in Construction (ITcon) 15(4), pp. 64-74.

Alicandro, M., Dominici, D. and Buscema, P. M., 2018. A new enhancement filtering approach for the automatic vector conversion of the UAV photogrammetry output. In: Euro-Mediterranean Conference, Springer, pp. 312-321.

Almac, U., Pekmezci, I. P. and Ahunbay, M., 2016. Numerical analysis of historic structural elements using $3 \mathrm{~d}$ point cloud data. The Open Construction and Building Technology Journal.

Angeli, S., Lingua, A. M., Maschio, P., Piantelli, L., Dugone, D. and Giorgis, M., 2018. Dense 3D model generation of a dam surface using UAV for visual inspection. In: International Conference on Robotics in Alpe-Adria Danube Region, Springer, pp. 151-162.

Armesto-González, J., Riveiro-Rodríguez, B., GonzálezAguilera, D. and Rivas-Brea, M. T., 2010. Terrestrial laser scanning intensity data applied to damage detection for historical buildings. Journal of Archaeological Science 37(12), pp. 30373047.

Bauer, E., Kraus, E., Silva, M. N. and Zanoni, V. A., 2014. Evaluation of damage of building facades in brasília. DBMC Durab Build Mater Compon 1, pp. 535-542.

Bosché, F. and Guenet, E., 2014. Automating surface flatness control using terrestrial laser scanning and building information models. Automation in construction 44, pp. 212-226.

Costantino, D. and Angelini, M., 2012. Process modeling and photogrammetric production for structural investigations concerning to the collapse of palazzo edilizia in Salerno (Italy). Lecture Notes in Computer Science (including subseries Lecture Notes in Artificial Intelligence and Lecture Notes in Bioinformatics) 7616 LNCS, pp. 440-448.

Galarreta, J. F., Kerle, N. and Gerke, M., 2015. Uav-based urban structural damage assessment using object-based image analysis and semantic reasoning. Natural Hazards and Earth System Sciences 15(6), pp. 1087.

Giacomucci, L., Bertoncello, R., Salvadori, O., Martini, I., Favaro, M., Villa, F., Sorlini, C. and Cappitelli, F., 2011. Microbial deterioration of artistic tiles from the façade of the grande albergo ausonia \& hungaria (venice, italy). Microbial ecology 62(2), pp. 287-298.

Guarnieri, A., Fissore, F., Masiero, A. and Vettore, A., 2017a. From TLS survey to 3D solid modeling for documentation of built heritage: The case study of porta Savonarola in Padua. ISPRS - International Archives of Photogrammetry, Remote Sensing and Spatial Information Sciences XLII-2/W5, pp. 303-308.

Guarnieri, A., Fissore, F., Masiero, A., Di Donna, A., Coppa, U. and Vettore, A., 2017b. From survey to FEM analysis for documentation of built heritage: the case study of villa RevedinBolasco. International Archives of the Photogrammetry, Remote Sensing and Spatial Information Sciences XLII-5/W1, pp. 527533.

Hällström, J., Barup, K., Grönlund, R., Johansson, A., Svanberg, S., Palombi, L., Lognoli, D., Raimondi, V., Cecchi, G. and Conti, C., 2009. Documentation of soiled and biodeteriorated facades: A case study on the coliseum, rome, using hyperspectral imaging fluorescence lidars. Journal of Cultural Heritage 10(1), pp. 106115. 
Hemmleb, M., Weritz, F., Schiemenz, A., Grote, A. and Maierhofer, C., 2006. Multi-spectral data acquisition and processing techniques for damage detection on building surfaces. International Archives of the Photogrammetry, Remote Sensing and Spatial Information Science.

Kim, M.-K., Sohn, H. and Chang, C.-C., 2014. Localization and quantification of concrete spalling defects using terrestrial laser scanning. Journal of Computing in Civil Engineering 29(6), pp. 04014086.

Maierhofer, C., Röllig, M., Steinfurth, H., Krankenhagen, R., Mecke, R., Schiller, M., Kernchen, A., Kalisch, U., Hennen, C., Meinhardt, J. et al., 2015. Detection and characterisation of safety relevant detachments of facade elements using a combination of active thermography and optical scanning methods. In: Proc. Of International Symposium Non-Destructive Testing in Civil Engineering(NDT-CE), http://www. ndt. net/search/link. php.

Masiero, A., Guarnieri, A., Pirotti, F. and Vettore, A., 2015. Semi-automated detection of surface degradation on bridges based on a level set method. ISPRS - International Archives of Photogrammetry, Remote Sensing and Spatial Information Sciences 40(3), pp. 15-21.

Pierdicca, R., Malinverni, E., Piccinini, F., Paolanti, M., Felicetti, A. and Zingaretti, P., 2018. Deep convolutional neural network for automatic detection of damaged photovoltaic cells. ISPRS International Archives of the Photogrammetry, Remote Sensing and Spatial Information Sciences XLII-2, pp. 893-900.
Sextos, A., De Risi, R., Pagliaroli, A., Foti, S., Passeri, F., Ausilio, E., Cairo, R., Capatti, M. C., Chiabrando, F., Chiaradonna, A. et al., 2018. Local site effects and incremental damage of buildings during the 2016 central italy earthquake sequence. Earthquake Spectra 34(4), pp. 1639-1669.

Simonyan, K. and Zisserman, A., 2014. Very deep convolutional networks for large-scale image recognition. arXiv preprint arXiv:1409.1556.

Sithole, G., 2008. Detection of bricks in a masonry wall. The International Archives of the Photogrammetry, Remote Sensing and Spatial Information Sciences pp. 1-6.

Suchocki, C. and Błaszczak-Bk, W., 2019. Down-sampling of point clouds for the technical diagnostics of buildings and structures. Geosciences.

Teza, G., Galgaro, A. and Moro, F., 2009. Contactless recognition of concrete surface damage from laser scanning and curvature computation. NDT \& E International 42(4), pp. 240-249.

Tucci, G., Bonora, V., Fiorini, L. and Conti, A., 2016. The Florence baptistery: 3-D survey as a knowledge tool for historical and structural investigations. ISPRS - International Archives of the Photogrammetry, Remote Sensing and Spatial Information Sciences 41, pp. 977-984.

Wang, Q., Kim, M.-K., Cheng, J. C. and Sohn, H., 2016. Automated quality assessment of precast concrete elements with geometry irregularities using terrestrial laser scanning. Automation in Construction 68, pp. 170-182. 